\title{
Comparison of Routing Protocol under Network Topology Change for Mobile Adhoc Network
}

\author{
Amit Dua, Rakesh Kumar Saini \\ Department of Computer Application, \\ DIT University, Dehradun, \\ Uttrakhand, India
}

\begin{abstract}
Mobile ad-hoc network (MANET) is an autonomous system of mobile nodes connected by wireless links. Each node operates as a router to forward packets and also an end system. The nodes moves free to organize themselves into a network. These node changes their position frequently. Special routing algorithm is needed to accommodate its changing topology. Flat routing protocols may be sufficient for small networks. Moreover either hierarchical or geographic routing protocols are needed in larger networks. Density, size and the mobility of the nodes are considered for choosing network protocols. In this Paper an rigorous attempt has been made for comparing the performance of two prominent distance vector routing protocols for MANETs: Destination Sequenced Distance Vector (DSDV) \& Dynamic Source Routing (DSR) routing protocols. As per our findings there is a significant performance differentials for both of these protocols.
\end{abstract}

\section{Keywords}

MANET, AODV, DSR, DSDV, Qualnet 5.0.2.

\section{INTRODUCTION}

Mobile devices, such as laptop computers, Pocket PCs, cellular phones, etc., are are becoming more popular in everyday life and are easily affordable, a[Johnson1994, Johnson1996]. At the same time, as the support for wireless networking products based on radio and infrared has been greatly increased over the past few years network connectivity options for mobile hosts have grown tremendously,. With the availability of mobile computing devices, mobile users have a Natural tendency to share information between them. even though there is no planning in advance and there is no Internet Connection available mobile users often want to have a meeting, For instance, there may be situations that employees find themselves together in a meeting room, or friends or business acquaintances may encounter each other in an airport terminal, or some scholars and researchers may meet in a hotel ballroom for a conference or workshop. In such situations, each user wants to connect to a wide-area network to communicate with each other may, this may not be convenient or practical because of the lack of Internet connectivity or because of the time or cost required for such a connection. In this Paper an attempt has been made to compare the performance of two prominent distance vector routing protocols for MANETs: Destination Sequenced Distance Vector (DSDV) \& Dynamic Source Routing (DSR) routing protocols. As per our findings the differences in the protocol mechanics lead to significant performance differentials for both of these protocols.

The performance differentials are analyzed using varying simulation time. These simulations are carried out using the
Qualnet 5.0.2. The results presented in this work illustrate the importance in carefully evaluating and implementing routing protocols in an ad- hoc environment. As of date, wireless communication is one of the most demanding areas of research within networking, with many proposed, but unverified protocols. The success of the proposed protocols depends on the availability of robust implementations that enable both real-time test beds and non-real time simulations. Routing in wireless ad-hoc networks is nontrivial due to highly dynamic environment. In recent years several routing protocols targeted at mobile ad-hoc networks are being proposed and prominent among them are DSDV and DSR. The performance comparison of these protocols considering all the characteristics that should be possessed by routing protocols is the fundamental step towards the invention of new routing protocol. This report does the detailed comprehensive analysis of routing protocols using Qualnet 5.0.2. All protocols are provided with identical traffic load and mobility patterns. In this comparison and performance evaluation analysis we try to judge the different important parameter like packet loss, packet delivery ratio, throughput, packet received etc. With the help of analysis result we can make sure that which routing protocol works better under different condition and whose QoS will be good for efficient communication for present and future application. Due to the restricted and limited bandwidth, it is vital that the mobile nodes make the most advantageous use of the connectivity when it arrives. Hence in order to select the data that need to be transmitted first, some sort of data prioritization is essential [3].

In addition, a simulation has been designed that guarantees enhanced accuracy and reduced delay. It is illustrated that our scenario reduces the packet drop increases quality of service by the extensive simulation result.

\section{MOBILE AD HOC NETWORK (MANET)}

Networks of nodes that just happen to be near each other are known as ad hoc networks or MANETs [4] (Mobile Ad hoc NETworks) ad hoc network is different from wired networks is that all the usual rules about fixed topologies, fixed and known neighbors, fixed relationship between IP address and location. Routers can come and go or appear in new places at the drop of a bit. With a wired network, if a router has a valid path to some destination, that path continues to be valid indefinitely. With an ad hoc network, the topology may be changing all the time, so desirability and even validity of path can change spontaneously, without warning. And such circumstances make routing in ad hoc networks different from routing in their fixed counter parts. 
Mobile Ad hoc Networks [5] enable host to maintain connectivity to the fixed network or exchange information when no infrastructure support, such as a base station or an access point, is available. This is generally achieved through multihop communication which allows a node to reach far away destinations by using intermediate nodes as relays. MANET routing protocols are subdivided into two categories as shown below:

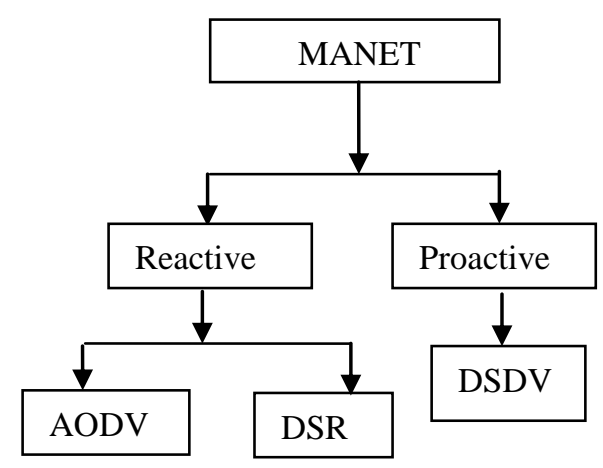

Fig.1.MANET and its concerned Routing Protocols

\section{REACTIVE ROUTING PROTOCOL}

These routing protocol are also called on demand [6] routing protocol since they do not maintain routing information or routing activity at the network nodes if there is no communication. If a node wants to send a packet to some another node then this protocol searches for the route in an on-demand manner and build the connection in order to transmit and receive the packet. The route discovery usually occurs by flooding the route request packets throughout the network.

\subsection{Ad-hoc On-demand Distance Vector Routing (AODV)}

AODV [7] uses routing tables, with one route entry per destination where each entry stores next hops towards destination. It broadcast route request (RREQ) packets and this RREQ [7] is uniquely identified by the sender address, destination address and request ID. If the node is either the destination node or has a route to the destination node then it returns a route reply (RREP) containing the route, to sender. AODV uses sequence numbers and node compares the destination sequence number of the RREQ with that of its route table entry this protocol either response with its own route if entry is fresh, or rebroadcasts the RREQ to its neighbors. In AODV [8], each node maintains a routing table which is used to store destination and next hop IP addresses as well as destination sequence numbers. And each entry in the routing table has a destination address, next hop, precursor nodes list, life time and distance to destination. Finally, after processing the RREP packet the node forwards it toward the source. The node can later update its routing information if it discovers a better path or route.

\subsection{Dynamic Source Routing (DSR) Protocol}

DSR [8] protocol is on demand which generally reduces the bandwidth especially in situations where the mobility is low. It is a simple and efficient routing protocol for using in ad-hoc networks this protocol has two important phases namely, route discovery and route maintenance. A node that desires communication with another node first searches its route cache to see if it already has a route discovery mechanism. This is done by sending Route Request message. When the node gets this message, it searches its own cache to see if it has a route to the destination. If it does not, it then appends its ID to the packet and forwards the packet to the next node. This process continuous until either a node with a route to the destination is encountered or the destination receives the packet. DSR support relatively rapid rates of mobility.

\subsection{Proactive Routing Protocol (Table- Driven Routing Protocol)}

In proactive routing, each node has one or more tables that consist of latest and update information of the routes to any node in the network. Each row has the next hop for reaching a node/subnet and the cost of this route. Various table-driven protocols differ in the way the information about a change in topology is propagated through all nodes in the networks. There exist some differences between the protocols that comes under this category depending on the routing information which is updated in each routing table. Also, these routing protocols maintain different number of tables. This protocol is not well node entries for each and every node in the routing table of every node this will cause more overhead in the routing table leading to more consumption of bandwidth. Example: Conventional routing schemes, DSDV.

\subsection{Destination-Sequenced Distance- Vectors Routing (DSDV) Protocol}

DSDV is a table-driven routing method for ad-hoc networks that is based on the Bellman-Ford algorithm. The main contribution of this algorithm was to sort out the Routing Loop problem which is present in Bellman-Ford algorithm[8]. And to do so, this protocol makes use of sequence numbers. Here each entry in the routing table contains a sequence number; the sequence numbers are generally Even if a link is present else, an Odd number is used. The number is generated by the destination, and the emitter needs to send out the next update with this number. Routing information is distributed between nodes by sending full dumps infrequently and smaller incremental updates more frequently.

\section{SCENARIO FOR PERFORMANCE ANALYSIS}

In this work 20 mobile nodes are considered, the initial position of these nodes are random in the flat grid aria of 800x800 and in z-direction 2000-3000 are considered, so nodes are spared in 800x800x1000 aria.

In this work one source and one destination node is considered, the starting node is the source node and last node is destination node. All node have mobility, in this work mobility is set as each node change its position after $10 \mathrm{sec}$. and after this node changes its position in every $5 \mathrm{sec}$. all new positions are random in nature. So in this work we compare 3 different routing protocols in the above scenario.

\section{SIMULATION TOOL AND ITS ENVIRONMENT}

The simulations where performed using Qualnet 5.0.2, which is particularly popular in the ad-hoc networking family. Qualnet 5.0.2 interprets the simulation scripts written c++ The user has to set the different components libraries up in the simulation environment. The user writes his simulation program as a c++ scripts. The main aim of choosing Qualnet 5.0.2 as a simulation tool among the other simulation tool because it supports networking research and education. It is 
also suitable for designing a new protocol, and comparing different protocol in different environment. Qualnet 5.0.2 is distributed freely and open source. A large number of institutes and people in development and research use, maintain and develop Qualnet 5.0.2, which increases the confidence in it. Qualnet 5.0.2also provides substantial support for simulation of TCP, UDP, routing and multicast protocol over wired and wireless network [10].

The traffic sources are CBR (Constant Bit-Rate). The sourcedestination is randomly spread over the network [9]. The mobility model uses ((RANDAM WAYPOINT MODEL)) in a rectangular aria of $800 \mathrm{~m} \times 800 \mathrm{~m}$ with 20 nodes. During the simulation starts its journey form a random spot to a random chosen destination and after every 5 second the topology of the network changed. Once the destination reached, the node takes a rest period of time in second and another random destination is chosen after that pause time the pause time is taken for this simulation is vary for $10 \mathrm{~s}, 20 \mathrm{~s}$ and $50 \mathrm{~s}$. this process repeats throughout the simulation, causing continuous changes in the topology of the underlying network [10][11]. Different network scenario for different number of nodes and pause time are generated. The modal parameters that have been used in the following experiments are summarized in Table 1.

Table 1. Simulation Parameters

\begin{tabular}{|c|c|}
\hline Parameter & Value \\
\hline Simulator & Qualnet 5.0.2 \\
\hline Protocols Considered & AODV, DSR \& DSDV \\
\hline Simulation Time & $10 \mathrm{~s}, 20 \mathrm{~s} \& 50 \mathrm{sec}$. \\
\hline Simulation Area & $800 \mathrm{~m} \times 800 \mathrm{~m}$ \\
\hline Transmission Range & $200-300 \mathrm{~m}$ \\
\hline Node Movement Model & Random Waypoint \\
\hline Bandwidth Used & 3 Mbps \\
\hline Traffic Type & CBR (TCP) \\
\hline Data Payload & Bytes/packet \\
\hline
\end{tabular}

\section{CALCULATION AND RESULT}

In this work three routing protocols has been considered that is two reactive type (DSR \& AODV) and one proactive type (DSDV) routing protocol. Finally we compare on the basis of Packet Delivery Fraction or Throughput, Packet loss, Routing load Fraction.

The calculation of one routing protocol (AODV) is described below and in similar fashion the remaining 2 routing protocol are calculated and analyzed.

\section{AODV (Reactive type):}

\section{Case I:}

Number of nodes considered $=20$

Pause time $=10$ sec.

Packet received will be $=4926$

Therefore original packet received $=4926-1235$ (Routing Packet $)=3691$

Packet sent will be $=4312$

Therefore Packet Delivery Fraction $(\mathrm{PDF})=85.59 \%$

And packet loss $=621$
Now sent routing packet $=612$

Received routing packet $=1235$

Therefore total routing packet $=1847$

Finally, routing load fraction will be $=37.49 \%$

Case II:

Number of nodes considered $=20$

Pause time $=20$ sec.

Packet received will be $=8725$

Therefore original packet received $=8725-2167$ (Routing Packet) $=6558$

Packet sent will be $=7768$

Therefore Packet Delivery Fraction $(\mathrm{PDF})=84.42 \%$

And packet loss $=1210$

Now sent routing packet $=1305$

Received routing packet $=2167$

Therefore total routing packet $=3472$

Finally, routing load fraction will be $=39.97 \%$

Case III:

Number of nodes considered $=20$

Pause time $=50$ sec.

Packet received will be $=32,130$

Therefore original packet received $=32,130-9806$ (Routing Packet $)=22,324$

Packet sent will be $=24515$

Therefore Packet Delivery Fraction $(\mathrm{PDF})=91.06 \%$

And packet loss $=2191$

Now sent routing packet $=4149$

Received routing packet $=9806$

Therefore total routing packet $=13955$

Finally, routing load fraction will be $=43.43 \%$

\section{SIMULATION RESULT}

For each simulation, we collect data about number of packets being dropped when the link breaks due to random motion. We also collected information about packet delivery ratio, packet loss and routing load fraction. And the simulation result is achieved from the trace file made by Qualnet 5.0.2 that report the time for each event in simulation model [12].

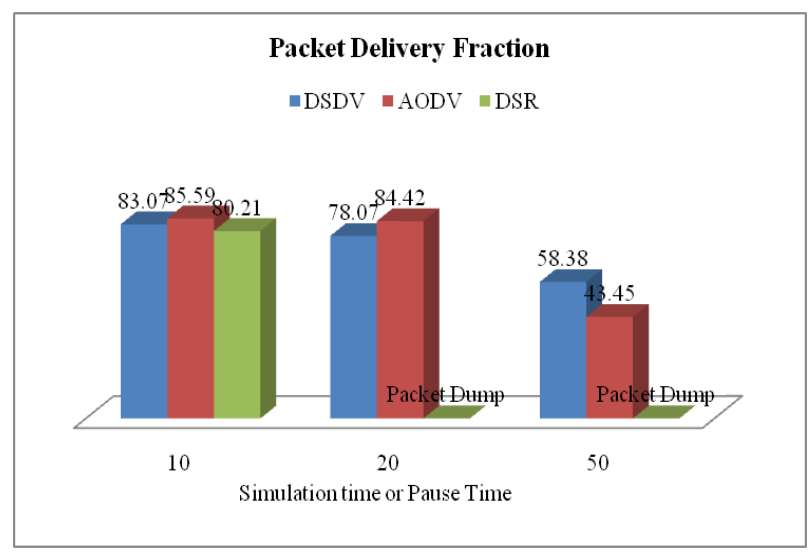

Fig.2 Packet Delivery Fraction 
(Figure 2) shows the packet delivery ratio for different routing protocol and depending on which link that breaks for pause time $10 \mathrm{sec}$. AODV has highest packet delivery fraction compare to other routing protocol. For pause time $20 \mathrm{sec}$ again AODV shows good performance compare to other protocol finally, for pause time $50 \mathrm{sec}$. DSDV has much better throughput compare to other routing protocol.

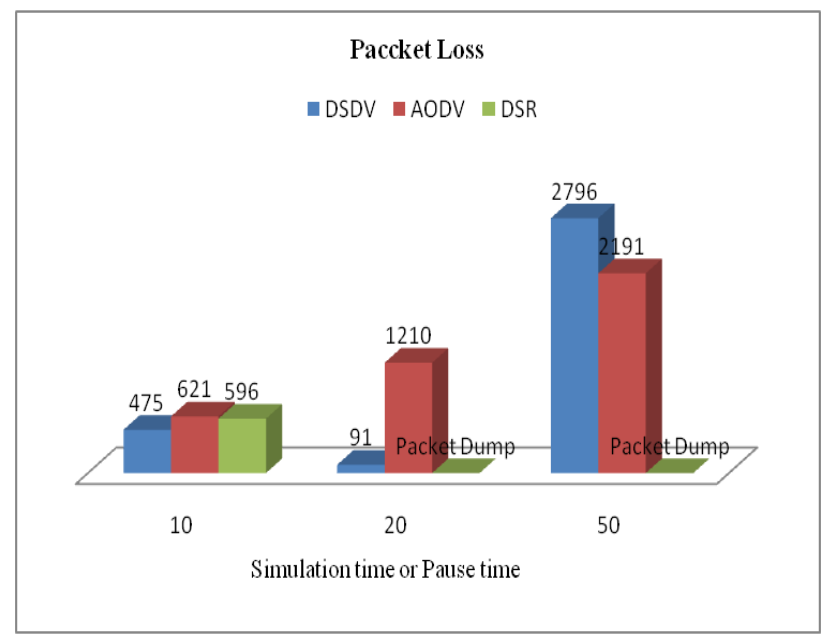

Fig. 3 Packet Loss

(Figure 3) shows the packet loss for different routing protocols in different pause times, for pause time $10 \mathrm{sec}$. DSDV has lowest packet loss compare to other routing protocols. For pause time 20sec AODV has the highest packet drop and DSDV has minimum packet drop. For 50sec. the packet drop will be much higher in DSDV compare to other routing protocol. DSR protocol gives core dumped result for $20 \& 50 \mathrm{sec}$ because of time out.

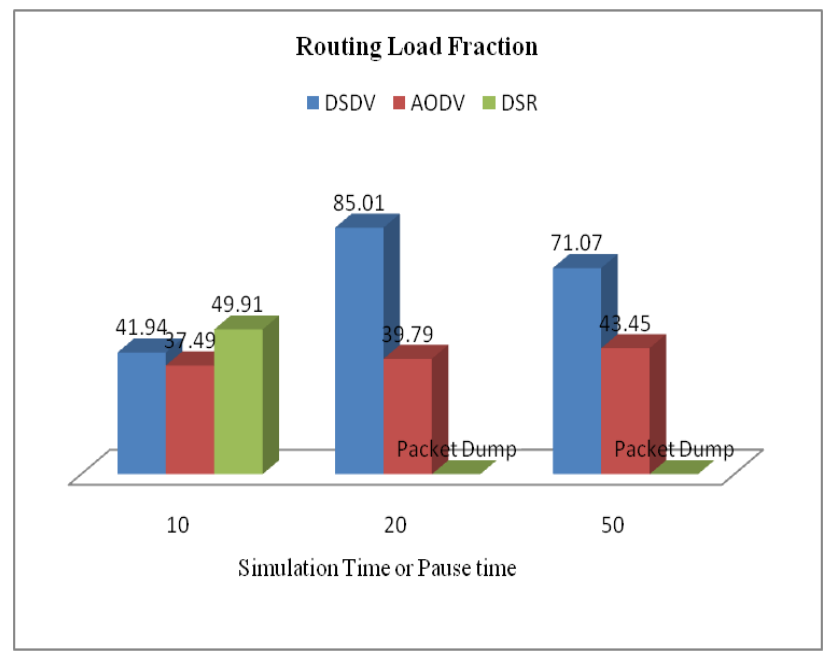

Fig. 4 Routing Load Fraction

(Figure 4) show the routing load fraction in different pause times for different routing protocols. For pause time $10 \mathrm{sec}$. the routing load fraction of DSR is highest compare to other routing protocols. For pause time $20 \mathrm{sec}$ the DSDV has the highest routing load fraction. Finally for pause time $50 \mathrm{sec}$. again DSDV has highest routing load fraction compared to other routing protocols. DSR give core dump result due to drop-tail.

\section{CONCLUSION}

From above simulation, result and calculation in different network environments that uses different topologies we conclude that the packet delivery fraction is more efficient for DSDV in different pause time. The packet loss will be much higher for DSDV when pause time increases. Finally we conclude for routing load fraction AODV proves to be more efficient and better than other routing protocol. The entire scenario in this simulation has been considered with our own consideration. Network security is the challenging issue which is our further work.

\section{REFERENCES}

[1] Toh, C.K. Delwar, M.Allen, "Evaluating the communication performance of an ad hoc wireless network", Wireless Communications, IEEE Transactions , vol.1, no.3, pp.402-414, Jul 2008.

[2] J Raju and J. J. Garcia-Luna-Aceves, "A comparison of on-demand and table driven routing for ad-hoc wireless networks," in IEEE International Conference on Communications, vol. 3, pp. 1702-1706, 2000.

[3] J. Broch, D. Maltz, D. Johnson, Y. Hu, and J. Jetcheva, "A performance comparison of multi-hop wireless ad hoc network routing protocols," in ACM/IEEE International Conference on Mobile Computing and Networking, vol.8, pp. 85-97, 1998.

[4] Josh Broch, David B. Johnsson, David A. Maltz, "The Dynamic Source Routing Protocol for Mobile Ad Hoc Networks", pp-19-23, October 1999.

[5] C.K. Toh, "A Novel Distributed Routing Protocol to Support Ad-Hoc Mobile Computing", Proc. 1996 IEEE 15th Annual Int'l. Phoenix Conf.Comp. and Communication., pp. 480-86, Mar. 1996.

[6] C. Hedrick, "Routing Information Protocol (RIP)", IETF: The Internet Engineering Taskforce, vol. 7, pp. 234-242, Jan 2006.

[7] J. Moy, "Open Shortest Path First (OSPF) Version 2", IETF: The Internet Engineering Taskforce, vol. 5, pp. 23 28, Apr 1998, 2006.

[8] B Williams. and T Camp, "Comparison of broadcasting techniques for mobile ad hoc networks", Proceedings of the ACM International Symposium on Mobile Ad Hoc Networking and Computing (MOBIHOC), pp.194-205, 2002.

[9] Sung-Ju Lee, Mario Gerla, and Ching-Chuan Chiang, "On-Demand Multicast Routing Protocol", IEEE Wireless Communications and Networking Conference (WCNC '99), pp-86. Sep 21-24, 1999.

[10] Y. Chun, L. Qin, L. Young and S. MeiLin, "Routing Protocols Overview and Design Issues for SelfOrganized Network", Proceedings of ICCT2000, pp. 1298-1303, Aug 2000.

[11] S.J. Lee, "Routing and Multicasting Strategies in Wireless Mobile Ad hoc Networks", Ph.D dissertation, University of California, Los Angeles, pp. 56-67, 2000.

[12] S. J. Lee, W. Su, J. Hsu, M. Gerla and R. Bagrodia, "A Performance Comparison Study of Ad Hoc Wireless Multicast Protocols", Proceedings of IEEE INFOCOM 2000, vol. 2 pp. 565-574, Mar 2000. 